\title{
Temperature Surges in Current-Limiting Circuit Devices
}

\author{
A. C. Fowler; I. Frigaard; S. D. Howison
}

SIAM Journal on Applied Mathematics, Vol. 52, No. 4. (Aug., 1992), pp. 998-1011.

Stable URL:

http://links.jstor.org/sici?sici=0036-1399\%28199208\%2952\%3A4\%3C998\%3ATSICCD\%3E2.0.CO\%3B2-N

SIAM Journal on Applied Mathematics is currently published by Society for Industrial and Applied Mathematics.

Your use of the JSTOR archive indicates your acceptance of JSTOR's Terms and Conditions of Use, available at

http://www.jstor.org/about/terms.html. JSTOR's Terms and Conditions of Use provides, in part, that unless you have obtained prior permission, you may not download an entire issue of a journal or multiple copies of articles, and you may use content in the JSTOR archive only for your personal, non-commercial use.

Please contact the publisher regarding any further use of this work. Publisher contact information may be obtained at http://www.jstor.org/journals/siam.html.

Each copy of any part of a JSTOR transmission must contain the same copyright notice that appears on the screen or printed page of such transmission.

The JSTOR Archive is a trusted digital repository providing for long-term preservation and access to leading academic journals and scholarly literature from around the world. The Archive is supported by libraries, scholarly societies, publishers, and foundations. It is an initiative of JSTOR, a not-for-profit organization with a mission to help the scholarly community take advantage of advances in technology. For more information regarding JSTOR, please contact support@jstor.org. 


\title{
TEMPERATURE SURGES IN CURRENT-LIMITING CIRCUIT DEVICES*
}

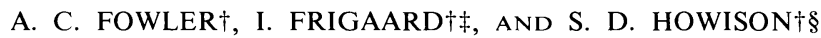

\begin{abstract}
This paper studies the problem of heat transfer in a thermistor, which is used as a switching device in electronic circuits. The temperature field is coupled to the current flow by ohmic heating in the device, and the problem is rendered highly nonlinear by a very rapid variation of electrical conductivity with temperature. Approximate methods based on high activation energy asymptotics are developed to describe the transient heat flow, which occurs when the circuit is switched on. In particular, it is found that a transient "surge" phenomenon (akin to thermal runaway, but self-saturating) occurs, and we conjecture that this phenomenon may be associated with cracking of thermistors, which sometimes occurs during operation.
\end{abstract}

Key words. thermal runaway, thermistor, Joule heating, high activation energy asymptotics

AMS(MOS) subject classification. 80A99

1. Introduction. This paper is concerned with heat and current flow in a circuit device called a thermistor. In appearance, this is a cylinder of typical radius $5 \mathrm{~mm}$ and typical thickness $2 \mathrm{~mm}$, connected into its circuit via wires soldered to the top and bottom; these surfaces are covered with a thin conducting sheet of metal acting as a contact (see Fig. 1).

The essential feature of a thermistor is that it is made from a ceramic material whose electrical conductivity varies strongly with temperature. There are two kinds: negative temperature characteristic (NTC) thermistors, whose electrical conductivity $\sigma$ increases with temperature $T$, and positive temperature characteristic (PTC) thermistors, for which $\sigma$ decreases with $T$ [1]; we discuss only the latter. (Here, positive and negative refer to materials whose resistivity is an increasing or decreasing function of temperature, respectively; conductivity is the inverse of resistivity.) The decrease in $\sigma$ is rapid, with a typical change being four orders of magnitude as $T$ increases from $100^{\circ} \mathrm{C}$ to $200^{\circ} \mathrm{C}$ (see Fig. 2).

There are many applications of thermistors; here we discuss their use as a fuse. In the circuit of Fig. 3, a short circuit is represented by closing the switch $S$, causing a current surge driven by the external voltage $V_{0}$ to pass through the circuit resistance $R_{0}$ and the thermistor, thereby heating it. The consequent decrease in the electrical conductivity causes the current to fall until equilibrium is reached, with all the heat generated within the thermistor being lost to its surroundings. In a well-designed thermistor, the final current should be a small fraction of the initial surge.

There are several problems of practical interest. First, it is often required to "tailor" a thermistor to a particular desired set of response characteristics, such as the switching time (the time taken for the current to fall to $1 / e$ times its initial value) and the final current. It is of interest to determine how these characteristics depend on design parameters such as, for example, size, aspect ratio, surface heat transfer, external resistance. Second, if $V_{0}$ is too large, the thermistor can crack. It is suspected that this cracking is caused by thermal stresses, and it is therefore important to find where large temperature gradients may occur.

\footnotetext{
* Received by the editors May 21, 1990; accepted for publication (in revised form) June 24, 1991.

† Mathematical Institute, Oxford University, 24-29 St. Giles', Oxford OX1 3LB, England.

¥ This author acknowledges an Advanced Studentship from the Science and Engineering Research Council.

$\S$ This author received support from the Royal Society via a University Research Fellowship.
} 


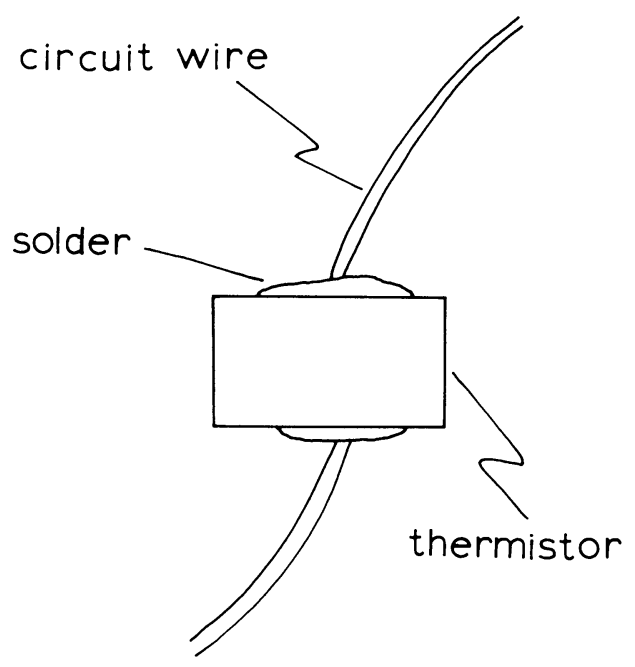

FIG. 1. A thermistor.

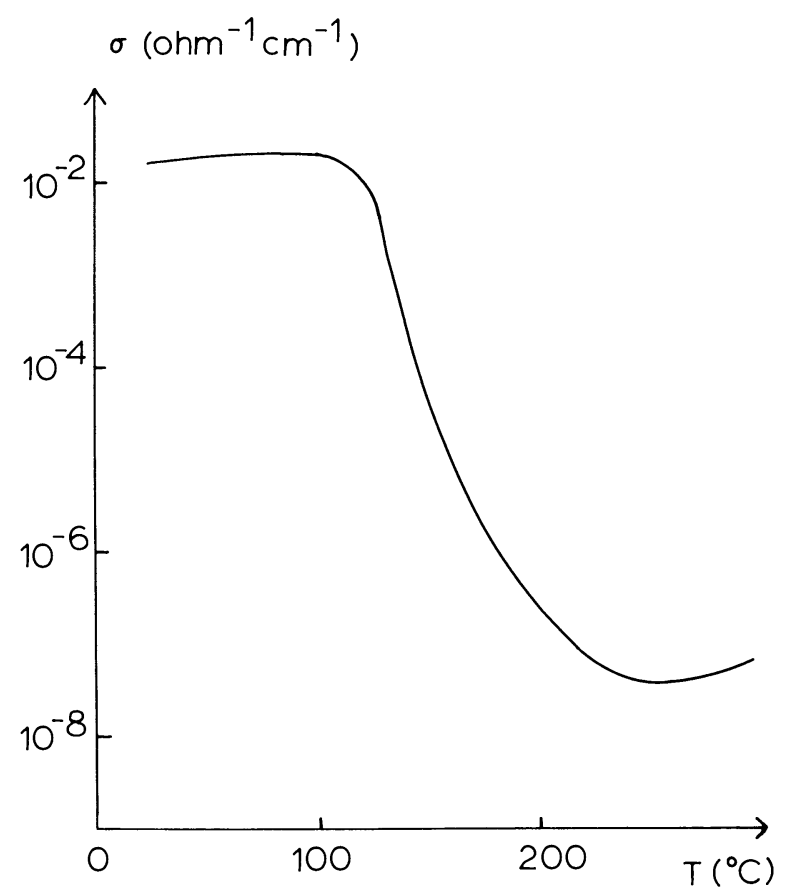

FIG. 2. Typical variation of conductivity with temperature for a PTC thermistor. The temperature where $\sigma$ is half its maximum value is designated the switching temperature. The diagram also gives an upside-down representation of $f(u)$ (see (2.5)).

The first step towards an answer to these questions is to set up a model for the heat and current flow in the thermistor, coupled with the current flow in the circuit. This we now do; in $\S \S 2-4$ we analyse it in the parameter regime of interest and describe a numerical solution, and in $\S 5$ (Conclusion) we summarise our results and relate them to other work on the problem. 


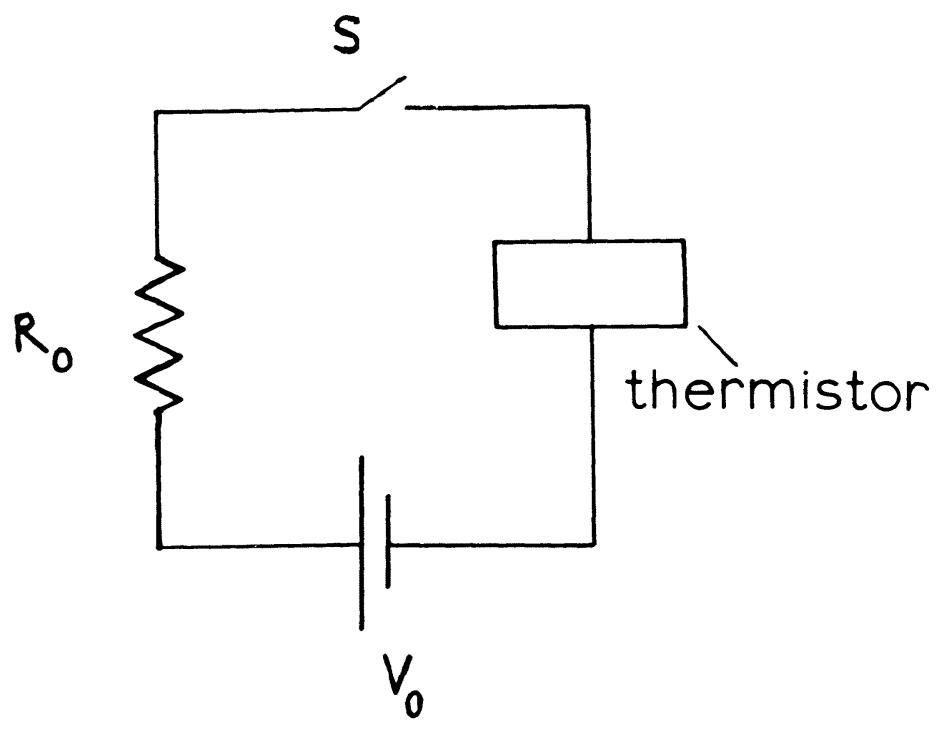

FIG. 3. Electrical circuit configuration.

Let $\varphi(\mathbf{x}, t)$ and $T(\mathbf{x}, t)$ be the electric potential and temperature, respectively, and $\sigma(T)$ the electrical conductivity. Then, in the thermistor $-H<z<H, 0 \leqq r<r_{0}, \varphi$ and $T$ satisfy

$$
\begin{gathered}
\nabla \cdot(\sigma(T) \nabla \varphi)=0, \\
\rho c \frac{\partial T}{\partial t}=k \nabla^{2} T+\sigma(T)|\nabla \varphi|^{2},
\end{gathered}
$$

where $\rho, c, k$ are the density, specific heat, and thermal conductivity, respectively, and $r, z$ are cylindrical polar coordinates. Equation (1.1) represents conservation of charge (the current density is $-\sigma(T) \nabla \varphi$ ), and the last term in (1.2) is the Joule heating caused by the current.

As boundary conditions for $T$ and $\varphi$, we take

$$
\begin{aligned}
& \varphi= \pm \varphi_{0} \quad \text { on } \quad z= \pm H, 0 \leqq r<r_{0}, \\
& \frac{\partial \varphi}{\partial r}=0 \quad \text { on } \quad r=r_{0},-H<z<H,
\end{aligned}
$$

with

$$
k \frac{\partial T}{\partial n}+h(\mathbf{x})\left(T-T_{a}\right)=0
$$

on all the boundary. Here $\varphi_{0}$ is to be determined from the circuit equation, and the whole top and bottom surfaces are equipotentials by virtue of the thin metal layer on them; $T_{a}$ is the ambient temperature and $h(\mathbf{x})$ is a heat transfer coefficient that will be different on different parts of the thermistor. In particular, the heat transfer will be greater to the solder than to the air.

Finally, we use Kirchhoff's law for the circuit: if $I(t)$ is the current and there are no capacitances or inductances, then

$$
V_{0}=2 \varphi_{0}+I R_{0} .
$$


However, we also have

$$
I=\left.2 \pi \int_{0}^{r_{0}}\left(\sigma(T) \frac{\partial \varphi}{\partial z}\right)\right|_{z=H} r d r,
$$

which follows from (1.1) and the definition of the current; so, eliminating $I$ and $\varphi_{0}$, (1.3) can be written as

$$
\varphi= \pm \frac{1}{2}\left(V_{0}-\left.2 \pi R_{0} \int_{0}^{r_{0}}\left(\sigma(T) \frac{\partial \varphi}{\partial z}\right)\right|_{z=H} r d r\right) \quad \text { on } z= \pm H .
$$

Equations (1.1), (1.2), (1.4), (1.5), and (1.8) constitute the dimensional model for the heat and current flow within the thermistor.

2. Nondimensionalisation. We nondimensionalise the model given in $\S 1$ by scaling the variables as follows:

$$
\begin{gathered}
\varphi=\left(V_{0} / 2\right) \bar{\varphi}, \quad T-T_{a}=(\Delta T) \bar{u}, \quad \mathbf{x}=H \overline{\mathbf{x}}, \\
t=\left(H^{2} \rho c / k\right) \bar{t}, \quad \sigma=\sigma_{U} \bar{\sigma}(\bar{u}) .
\end{gathered}
$$

Here $T_{a}$ is the ambient (e.g., room) temperature, and if $T_{s}$ is the temperature at which $\sigma$ first decreases (see Fig. 2), then $\Delta T=T_{s}-T_{a} \sim 100 \mathrm{~K}$ is typical both of the temperature range over which the conductivity drops and of the temperature difference between the hot thermistor and its environment; $\sigma_{U}$ is the ambient (room temperature) conductivity. In terms of these scales, the corresponding dimensionless version of the model, dropping the overbars, is

$$
\begin{gathered}
\nabla \cdot[\sigma \nabla \varphi]=0, \\
u_{t}=\nabla^{2} u+\gamma \sigma|\nabla \varphi|^{2},
\end{gathered}
$$

with

$$
\varphi= \pm\left[1-\left(2 \mu / a^{2}\right) \int_{0}^{a} \sigma \frac{\partial \varphi}{\partial z} r d r\right] \text { on } z= \pm 1 \text {, }
$$

$$
\frac{\partial \varphi}{\partial r}=0 \quad \text { on } r=a
$$

$$
\frac{\partial u}{\partial n}+\beta u=0 \quad \text { on } z= \pm 1, r=a
$$

where

$$
a=r_{0} / H, \quad \beta=h H / k, \quad \mu=\pi H R_{0} \sigma_{U} a^{2} / 2, \quad \gamma=\sigma_{U} V_{0}^{2} / 4 k \Delta T .
$$

The variation of $\sigma$ with $u$ given in Fig. 2 can be represented as

$$
\sigma=\exp [-f(u) / \varepsilon]
$$

where the data suggests $\varepsilon \sim 10^{-1}$. For example, we could choose

$$
f(u)= \begin{cases}0, & u<1, \\ u-1, & 1<u<2 \\ 1, & u>2\end{cases}
$$

as a crude representation (see $\S 5$ ). 
Typical values of the parameters are

$$
\begin{aligned}
\rho & =5.6 \times 10^{3} \mathrm{~kg} \mathrm{~m}^{-3}, & c & =540 \mathrm{~J} \mathrm{~kg}^{-1} \mathrm{~K}^{-1}, \\
k & =2 \mathrm{~W} \mathrm{~K}^{-1} \mathrm{~m}^{-1}, & \sigma_{U} & =2 \mathrm{~m}^{-1} \Omega^{-1}, \\
R_{0} & =50-500 \Omega, & V_{0} & =250 \mathrm{~V}, \\
r_{0} & =5 \times 10^{-3} \mathrm{~m}, & H & =10^{-3} \mathrm{~m}, \\
\Delta T & =100 \mathrm{~K} . & &
\end{aligned}
$$

Values of $h$ are less certain, but we might take

$$
\begin{aligned}
& h \sim 10^{2} \mathrm{~W} \mathrm{~m}^{-2} \mathrm{~K}^{-1} \text { on thermistor/solder interfaces, } \\
& h \sim 10 \mathrm{~W} \mathrm{~m}^{-2} \mathrm{~K}^{-1} \text { on thermistor/air interfaces }
\end{aligned}
$$

(a typical average measured value is $40 \mathrm{~W} \mathrm{~m}^{-2} \mathrm{~K}^{-1}$, but the solder is much more conductive than air). With these values, we find that

$$
a \sim 5, \quad \mu \sim 4-40, \quad \gamma \sim 150, \quad \beta \sim 10^{-1} \text { (top) }-10^{-2} \text { (sides). }
$$

One important feature is the large value of $\gamma$. While $\sigma \sim 1$, this causes rapid ohmic heating of the whole device to occur. As $u$ increases through the switching temperature, $\sigma$ drops rapidly, and we can expect the temperature to reach equilibrium after a further rise. Our aim is to find this limiting temperature and the consequent current.

Several authors have worked on versions of the thermistor problem. There is general agreement that the field equations are (2.2), but for various reasons (including mathematical convenience), the boundary conditions (2.3) differ with each author. In particular, many previous treatments have taken $\mu=0$. We always assume that $\mu>0$, and defer until $\S 5$ a review of other work.

3. "Large activation energy asymptotics": One-dimensional analysis. Since the heat transfer coefficient is very small $\left(\beta \sim 10^{-2}\right)$ at the sides, we expect that the temperature and potential fields will be approximately one-dimensional. This is accentuated by the relatively large aspect ratio $(a \approx 5)$, and will be a uniformly accurate approximation since we have derivative boundary conditions at the sides. We thus seek solutions as functions of $z$ and $t$ only. It then follows from (2.2a) that

$$
\sigma \frac{\partial \phi}{\partial z}=C(t)
$$

and application of (2.3a) together with (2.5) leads to the following problem for the temperature field $u$ :

$$
\begin{aligned}
& \phi=C \int_{0}^{z} \exp [f(u) / \varepsilon] d z, \\
& C=\left[\mu+\int_{0}^{1} \exp [f(u) / \varepsilon] d z\right]^{-1}, \\
& u_{t}=u_{z z}+\gamma C^{2} \exp [f(u) / \varepsilon] .
\end{aligned}
$$

When the device is operating satisfactorily, and with a function $f(u)$ such as that in (2.6), we can expect that the steady maximum temperature $u^{*}$ is at $z=0$, and that $u^{*}>1$. In a steady state, we expect a thin hot region near $z=0$ where $u \approx u^{*}$, the maximum value of $u$, and a thick "warm" region where $1 \leqq u<u^{*}$. ("Cold" will mean $u<1$.) The value of $u^{*}$ is to be found. 
We define

$$
\Delta=\exp \left[f\left(u^{*}\right) / \varepsilon\right] \gg 1
$$

so that $u$ satisfies

$$
u_{t}=u_{z z}+\frac{\gamma \Delta \exp \left[\left\{f(u)-f\left(u^{*}\right)\right\} / \varepsilon\right]}{\left[\mu+\Delta \int_{0}^{1} \exp \left[\left\{f(u)-f\left(u^{*}\right)\right\} / \varepsilon\right] d z\right]^{2}} .
$$

The precepts of large activation energy asymptotics are based on solving (3.4) in the limit as $\varepsilon \rightarrow 0$. We note that there are other large and small parameters $\gamma, \mu, \beta$ in the problem, and, to be specific, we conduct the initial discussion on the basis that these are all $O(1)$. Further simplifications may ensue if further approximations are made (such as that $\beta \ll 1$ ): these can be taken as special cases of our initial assumptions.

Steady state. Experience with large activation energy asymptotics suggests that in a steady state there will be a thin hot region where $u-u^{*} \sim \varepsilon$, and thus $z \sim \varepsilon$, also.

In the hot region, put

$$
z=\varepsilon \zeta, \quad u=u^{*}+\varepsilon w / f^{\prime}\left(u^{*}\right),
$$

so that, to leading order, (3.4) is (with $\partial / \partial t=0$ )

$$
0=w_{\zeta \zeta}+e^{w} /\left(\left(\mu / \gamma f^{\prime}\left(u^{*}\right)\right)+\int_{0}^{\infty} e^{w} d \zeta\right)^{2},
$$

provided that we choose

$$
\Delta=\gamma f^{\prime}\left(u^{*}\right) / \varepsilon,
$$

and assuming that $\mu \leqq \gamma$. This is reminiscent of a problem treated by Pearson [14] (see also Ockendon and Ockendon [13]) in variable viscosity shear flow. Equation (3.7) implies that the maximum temperature $u^{*}$ is given by

$$
u^{*} \approx f^{-1}[\varepsilon \ln (\gamma / \varepsilon)]
$$

for example, the exponential law (2.6) gives us

$$
u^{*} \approx 1+\varepsilon \ln (\gamma / \varepsilon) \text {. }
$$

The outer problem $(z \sim O(1))$ is purely conductive, since the ohmic heating term there is $\varepsilon^{-1} \exp [-O(1 / \varepsilon)] \ll 1$.

Transient. The transient approach to the steady state may be described in a similar way. We must solve (3.4), i.e.,

$$
u_{t}=u_{z z}+\frac{\gamma \exp [f(u) / \varepsilon]}{\left[\mu+\int_{0}^{1} \exp [f(u) / \varepsilon] d z\right]^{2}},
$$

with $u_{z}=0$ on $z=0, u_{z}+\beta u=0$ on $z=1$. This is an interesting problem in its own right and bears some similarity to problems studied by Lacey [10]. Here we sketch a possible scenario.

There is an initial "warm-up" period while $u<1$, when $\sigma=1$, and $u$ satisfies

$$
u_{t}=u_{z z}+\frac{\gamma}{(1+\mu)^{2}}
$$

for the values of $\gamma, \mu$, and $\beta$ that we are considering, the maximum temperature at the centre will reach 1 in finite time, and, following this, there is a secondary "surge" 
phase when the temperature increases rapidly. Define the function $v(u)$ and the fast timescale $\tau$ by

$$
f(u)=\varepsilon v, \quad t=\varepsilon \mu^{2} \gamma^{-1} \tau ;
$$

then where $f^{\prime}>0$, i.e., $v>0(u>1), v$ satisfies

$$
v_{\tau}=\frac{f^{\prime}(u) e^{v}}{\left[1+\mu^{-1} \int_{0}^{1} e^{v} d z\right]^{2}}+\frac{\varepsilon \mu^{2}}{\gamma} v_{z z}-\frac{\varepsilon^{2} \mu^{2}}{\gamma} \frac{f^{\prime \prime}}{f^{\prime 2}} v_{z}^{2} ;
$$

at leading order, this gives, at least while $v$ is $O(1)$,

$$
v_{\tau} \approx f^{\prime}(u) e^{v} /\left[1+\mu^{-1} \int_{0}^{1} e^{v} d z\right]^{2} .
$$

Thus $v$ begins to increase on a fast timescale $t \sim \varepsilon \mu^{2} / \gamma$.

Note that the warm-up occurs (from (3.11)) on a timescale $t \sim \mu^{2} / \gamma$; and since (3.14) applies where $v>0$, the rapid surge phase is initially confined to the central region where $u \approx 1$; thus $\int_{0}^{1} e^{v} d z \approx 1$ (since $v=0$ for $u<1$ ), and $v_{\tau} \approx f^{\prime} e^{v} /\left(1+\mu^{-1}\right)^{2}$. Since $u$ is a slowly varying function of $v$ (and hence $\tau$ ), so is $f^{\prime}(u)=f^{\prime}\left[f^{-1}(\varepsilon v)\right]$, we can solve this approximately by taking $f^{\prime}$ as a constant, whence

$$
v \approx-\ln \left[e^{-v_{0}(z)}-f^{\prime} \tau /\left(1+\mu^{-1}\right)^{2}\right] .
$$

The solution (3.15) leads to blowup in finite time. This is rapidly suppressed as $v$ increases, because the integral term becomes important. Returning to the original timescale, (3.13) is

$$
v_{t}=v_{z z}-\varepsilon\left(f^{\prime \prime} / f^{\prime 2}\right) v_{z}^{2}+(\gamma / \varepsilon) f^{\prime} e^{v} /\left[\mu+\int_{0}^{1} e^{v} d z\right]^{2} .
$$

Saturation of $v$ occurs when $v \approx \ln (\gamma / \varepsilon)$, since then the integral term becomes important. Putting

$$
v=\ln (\gamma / \varepsilon)+w,
$$

we have the approximate transient problem (for $w>-\ln (\gamma / \varepsilon)$ )

$$
w_{t}=w_{z z}+\frac{f^{\prime} e^{w}}{\left[\int_{0}^{1} e^{w} d z\right]^{2}},
$$

which relaxes towards the previously defined steady state on a conductive timescale $O(1)$.

In fact, this final conductive relaxation will generally take place in two stages, depending on how uniform the initial warm-up period is. In general, the central portion surges for a time $t \sim \varepsilon \mu^{2} / \gamma$; during this time the cold $(u<1)$ region suffers an increase of $\Delta u \sim \varepsilon$. If $u_{z z} \sim O(1)$ at $z=0$ when $u=1$, then the surge propagates out to $z=O(\sqrt{\varepsilon})$ (i.e., where $1-u \sim \varepsilon$ ). Rescaling $z \sim \varepsilon^{1 / 2}$ then suggests that the hot zone governed by (3.17) equilibrates in a time $t \sim \varepsilon$, and the outer temperature field relaxes conductively on a time $t=O(1)$. On the other hand, if $1-u \sim \varepsilon$ everywhere at surge initiation, then the whole thermistor participates in the surge, and surge termination and final relaxation both occur on a timescale $O(1)$. Such uniform initial temperatures can be caused by large numerical values of $\gamma / \mu^{2}$ or small values of $\beta$, such as we, in fact, have. To summarise, there are four distinct phases: warm-up $t \sim \mu^{2} / \gamma$; surge $t \sim \varepsilon \mu^{2} / \gamma$; deceleration $t<1$; and relaxation $t \sim 1$ : compare Fig. 4 .

In this description, then, the largest temperature gradients are set up towards the centre of the device, and, although further analysis of (3.13) is then necessary, (3.15) 
suggests that $\partial v / \partial z \approx e^{v} v_{0}^{\prime} \sim \mu$ when $e^{v} \sim \mu$. These large gradients occur in a "front" dividing the hot central surging region from the cooler periphery (see Fig. 4 below). Thus, surging leads to temperature gradients $\partial u / \partial z \sim \varepsilon \mu$, which are typically significantly larger than those associated with an almost uniform warm-up phase. Values $\varepsilon \mu$ of $O(1)(\varepsilon=0.1, \mu=10)$ correspond to temperature gradients of order $100^{\circ} \mathrm{C}$ per millimetre.

4. The case where $\beta \ll 1$, two dimensions, numerical results. The analysis given in the preceding section applies for $\varepsilon \ll 1, \beta \sim 1$. A particular further simplification follows if we suppose that $\beta \ll 1$, also, as is, in fact, the case $(\beta \approx 0.1$ on the top). In this case, the initial warm-up is close to uniform, as is the final steady state. Let us put

$$
\beta=\varepsilon b
$$

and write

$$
u=u^{*}+\varepsilon w / f^{\prime}\left(u^{*}\right)
$$

in the steady state. Define $\Delta$ as before, by (3.7). Then, if $\Delta \gg \mu$, i.e., $\gamma \gg \varepsilon \mu, w$ is given to leading order by the (unique) solution of

$$
w_{z z}+e^{w} /\left[\int_{0}^{1} e^{w} d z\right]^{2}=0 ; \quad w_{z}=-b f^{\prime}\left(u^{*}\right) u^{*} \quad \text { on } z=1 ; \quad w_{z}=0 \quad \text { on } z=0
$$

so that $u \approx u^{*}$ everywhere.

It is not so obvious whether $u \approx \bar{u}(t)$, where $\bar{u}$ is the average temperature, applies throughout the transient. To examine this, we formally write

$$
u=\bar{u}(t)+\varepsilon V, \quad t=\left(\mu^{2} \varepsilon / \gamma\right) \tau,
$$

and seek conditions under which $V$ is uniformly $O(1)$. Evidently, we can take $V=O(1)$ at most when $\bar{u}=1$ (at the end of the warm-up phase). During the acceleration phase, while the integral term in (3.10) is small, $\bar{u}$ must satisfy

$$
\frac{d \bar{u}}{d \tau} \approx \varepsilon \exp [f(\bar{u}) / \varepsilon]
$$

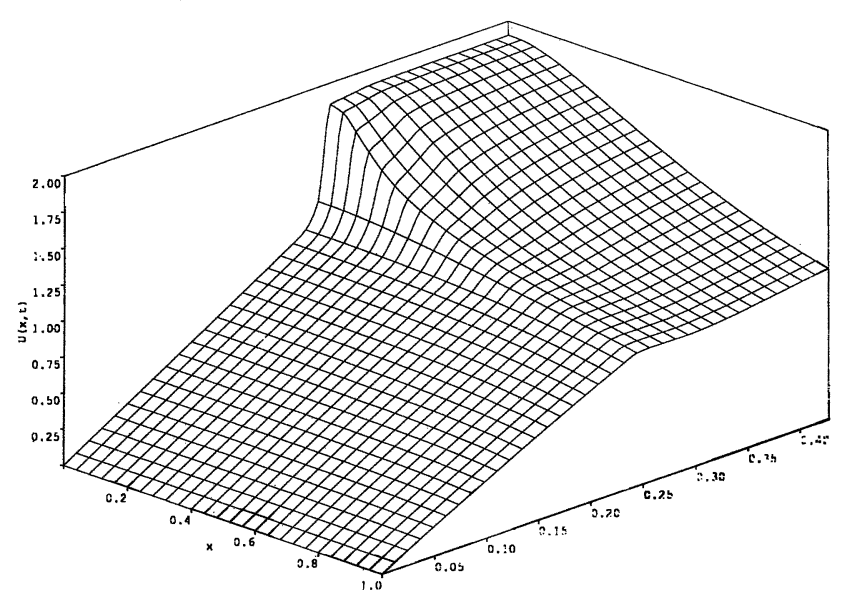

FIG. 4. Evolution temperature in the one-dimensional case. Parameter values are $\gamma=2000, \varepsilon=0.1, \mu=20$, $\beta=0.5$. 
by explicit calculation, $V$ satisfies

$$
\begin{aligned}
V_{\tau} & =\left(\mu^{2} \varepsilon / \gamma\right) V_{z z}+\frac{\bar{u}^{\prime}}{\varepsilon}\left[\frac{\exp [\{f(\bar{u}+\varepsilon V)-f(\bar{u})\} / \varepsilon]}{\left[1+\mu^{-1} \int_{0}^{1} \exp \{f(\bar{u}+\varepsilon V) / \varepsilon\} d z\right]^{2}}-1\right] \\
& \approx \frac{\bar{u}^{\prime}}{\varepsilon}\left[\exp \left\{f^{\prime}(\bar{u}) V\right\}-1\right],
\end{aligned}
$$

for sufficiently small values of $\tau$. Eliminating $\tau$, we then have $V$ given in terms of $\bar{u}$ by

$$
\frac{\partial V}{\partial \bar{u}}=\frac{1}{\varepsilon}\left[\exp \left\{f^{\prime}(\bar{u}) V\right\}-1\right],
$$

with $V=O(1)$ when $\bar{u}=1$. It follows from this that $V$ will become much larger than 1 for $\bar{u}-1=O(1)$. Moreover, spatial variations in $V$ at the onset of the acceleration phase will lead to large spatial gradients in $V$, and hence $u$, as $\bar{u}$ increases. In other words, we may expect the same surge structure to develop even when $\beta \ll 1$. Only if $u=1+O\left(\varepsilon^{2}\right)$ at surge onset will $u$ remain approximately uniform, since then (4.7) will imply that $V=O(1)$. Such uniform initial profiles are associated with large numerical values of $\gamma / \mu^{2}$.

It is easy to extend these results (when $\beta \ll 1$ ) to two dimensions, at least for the steady state. Returning to (2.2) and (2.3), we substitute for $u$ from (3.5); then choosing $\Delta$ given by (3.3) to satisfy (3.7), we find, to leading order, that

$$
\begin{gathered}
\boldsymbol{\nabla} \cdot\left[e^{-w} \nabla \varphi\right]=0, \\
\nabla^{2} w+e^{-w}|\nabla \varphi|^{2}=0,
\end{gathered}
$$

with

$$
\begin{aligned}
& \frac{\partial w}{\partial n} \approx-b f^{\prime}\left(u^{*}\right) u^{*} \quad \text { on } z=1, r=a ; \quad \frac{\partial w}{\partial n}=0 \quad \text { on } z=0 ; \\
& \frac{\partial \varphi}{\partial r}=0 \quad \text { on } r=a ; \quad \varphi=0 \quad \text { on } z=0 ; \quad \varphi \approx 1 \quad \text { on } z=1,
\end{aligned}
$$

as long as $\Delta \gg \mu$.

If $u$ is very uniform in the warm-up phase, then $u$ will remain uniform in the surge phase, and the evolution of the temperature can be obtained by integrating $(2.2 \mathrm{~b})$ over the volume of the thermistor. Using (2.2a) and (2.3c), we get

$$
\frac{d}{d t} \int_{V} u d V=-\int_{S} \beta u d S+\gamma \int_{S} \sigma(u) \phi \frac{\partial \varphi}{\partial n} d S .
$$

With $\beta \ll 1$, we may take $u \approx \bar{u}$, even in the boundary layer at $z=1$. On the other hand, if $u-\bar{u} \ll \varepsilon$ during the surge phase, then approximately $\nabla^{2} \varphi=0$, whence $\varphi_{z z}=0$, so that $\partial \varphi / \partial n=\varphi$ (=constant) on $z=1$. Hence (2.3a) implies that $\varphi \approx 1 /(1+\mu \sigma(u))$ on $z=1$, and this applies after the surge phase, also, when $\varphi \approx 1$. Hence

$$
\int_{S} \sigma(u) \phi \frac{\partial \varphi}{\partial n} d S=\int_{z=1} \sigma \varphi \frac{\partial \varphi}{\partial n} d S \approx \pi a^{2} \sigma /[1+\mu \sigma]^{2}
$$

This will not be the case in the conductive phase (where $\varphi$ satisfies (4.8a)), but the error is not serious. Hence a uniform approximation to (4.10) may be obtained by writing $u \approx \bar{u}(t)$, which satisfies

$$
V \frac{d \bar{u}}{d t}=-\bar{\beta} S \bar{u}+\gamma A \sigma(\bar{u}) /[1+\mu \sigma]^{2},
$$


where $V=\pi a^{2}$ is (half) the thermistor volume, $S=\pi a(2+a)$ is (half) the total external surface area, $\boldsymbol{A}=\pi a^{2}$ is the cross-sectional area, all in dimensionless units, and $\bar{\beta}$ is the mean value of $\beta$. Noting that $\beta=\varepsilon b$, the equilibrium value of $u^{*}$ is determined from

$$
\Delta=1 / \sigma\left(u^{*}\right) \approx \gamma A / \varepsilon \bar{b} S u^{*}
$$

$(\sigma \ll 1)$, which is consistent with (3.7) (to $O(\varepsilon)$ in $u^{*}$ ). Equation (4.12) provides a convenient means of estimating transient and steady behaviour, at least when $\beta$ is small. When $\beta \sim \varepsilon$, it only applies if $\gamma / \mu^{2} \gg 1$, so that $u$ is truly uniform; if $\gamma / \mu^{2} \sim 1$, we expect a surge to develop as previously described.

To illustrate these results, we solve the one-dimensional problem (3.3) numerically, for a variety of values of the parameters. Figure 4 illustrates a typical solution for values $\gamma=2000, \varepsilon=0.1, \mu=20$, and $\beta=0.5$. It can be seen that a central surge does indeed occur, as predicted here, and that its features are in line with the discussion in the text. In Fig. 5, we plot the numerically computed maximum temperature versus the asymptotic estimate. It can be seen that the prediction is excellent, and that the error is apparently of $O(\varepsilon)$, which is consistent with our analysis.

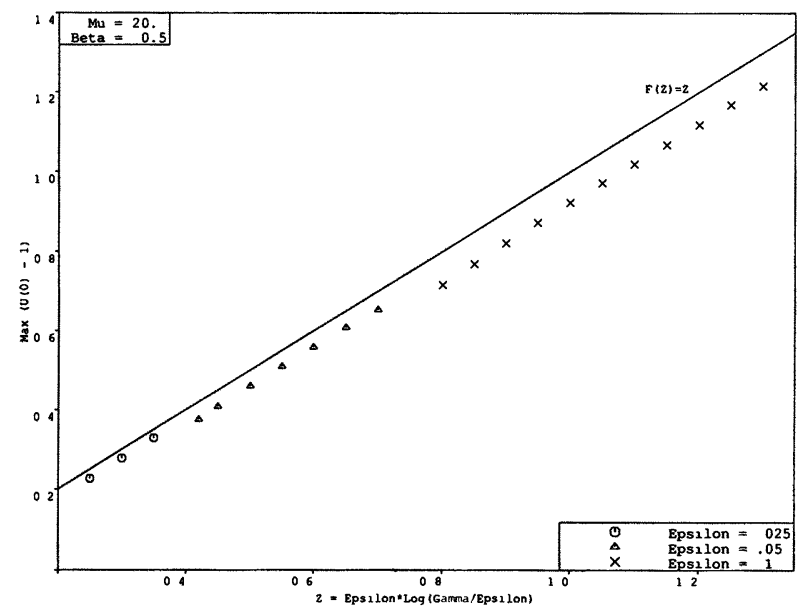

FIG. 5. Comparison of numerical values of $u^{*}-1$ with the theoretical prediction (4.8), for values of $\varepsilon=0.1$ (crosses), $\varepsilon=0.5$ (triangles), $\varepsilon=0.025$ (circles).

In Fig. 6, we show numerical solutions of (4.12), for values $a=5, \beta=0.1, \gamma=150$, and for various values of $\varepsilon$. The development of a surge phase as $\bar{u}$ increases through 1 is notable; while its duration decreases as $\varepsilon$ decreases, so does its amplitude, since $u^{*} \rightarrow 1$ as $\varepsilon \rightarrow 0$.

5. Conclusion. Early treatments of the thermistor problem ignored the interaction with the circuit by setting $\mu=0$ [11], [12]. The quadratic nonlinearity in the field equations has recently stimulated mathematical interest in this version of the problem, and results are available for various boundary conditions for $u$ and $\varphi$. Cimatti and Prodi [5] and Cimatti [2], [4] obtain existence and regularity results when $u$ and $\varphi$ have Dirichlet boundary data; i.e., both are specified on the thermistor boundary. Howison, Rodrigues, and Shillor [9] also obtain regularity results with the radiation condition (2.3c). Howison [7] discussed a simplified version in which $\varphi$ satisfies (2.3a), (2.3b) with $\mu=0$, but $u=0$ on the boundary (i.e., $\beta \rightarrow \infty$ ), and with $\sigma(u)$ represented by a step function as in $\S 3$. Howison concluded that as long as $\gamma$ is not too large, the 


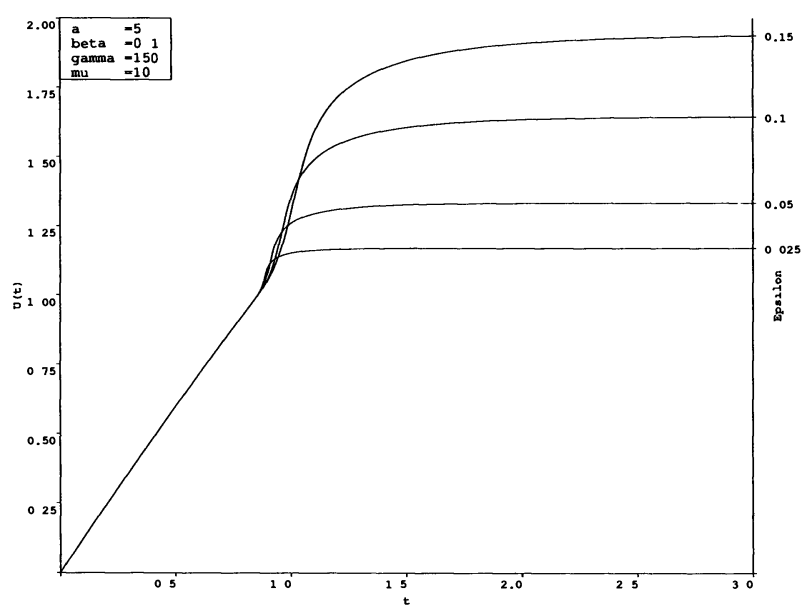

FIG. 6. Numerical solutions of (4.12), with $a=5, \beta=0.1, \gamma=150$, and various $\varepsilon$.

hot region $u>1$ must be thin $(O(\gamma \delta))$. This work was extended by Westbrook [15] to incorporate the more realistic boundary condition $(2.3 \mathrm{c})$; he presented numerical results for an asymptotic problem exploiting the thinness of the hot region. The limiting case where $\delta=0$ is an interesting unsolved problem.

A striking result due to Diesselhorst [6], revived and made rigorous by Cimatti [3], is that, no matter what shape the thermistor is, if $u$ and $\varphi$ satisfy boundary conditions such that on the portions of the boundary where $\varphi$ is constant, $u$ is constant also, and on the portions where $\partial \varphi / \partial n$ vanishes so does $\partial u / \partial n$, then the equipotentials and isotherms for the steady problem form the same family of curves, and the problem reduces to solving one nonlinear ordinary differential equation and Laplace's equation. Specifically, since $u=u(\varphi)$, we can write $\sigma(u) \nabla \varphi=\nabla \psi$ for some $\psi$, so that from (2.2a), $\nabla^{2} \psi=0$; then from $(2.2 \mathrm{~b})$, we find that $d / d \varphi((1 / \sigma(u) /(d u) d \varphi))=-1$. In two space dimensions, $\psi$ has a geometrical interpretation as the imaginary part of the conformal map from the thermistor to a rectangle [8]. These results for the steady state problem can be extended to include the effect of an external resistance, although, of course, in the steady state the resistance of the thermistor should be much larger than any external resistance.

Our results differ from these in two respects: first, we obtain asymptotic results for the unsteady case, and, second, we incorporate the electric circuit. Our conclusions may be summarised as follows.

1. The system we describe depends on five important dimensionless parameters: $\gamma, \mu, \varepsilon, \beta$, and $a$. Of these, $\mu$ and $a$ may be varied by changing the shape of the thermistor, $\beta$ depends on the exact mechanism of heat transfer away from the device, $\gamma$ depends only on $V_{0}$ and the intrinsic properties of the ceramic material (assuming that $\Delta T$ is more or less invariant), and $\varepsilon$ depends entirely on intrinsic material properties.

2. Provided that $\gamma$ is large enough that the switching temperature is easily reached, the largest dimensionless temperature is $u^{*} \sim 1+O(\varepsilon \ln (\gamma / \varepsilon))$, from (3.8). In particular, if $\beta$ and $\varepsilon$ are of similar size, then the steady temperature is $u^{*}+O(\varepsilon)$ throughout the device.

3. The current-voltage dependence in the steady state is easily calculated. We begin with the case in which $R_{0}=0$, and then we use (1.5) to calculate the steady current for $R_{0} \neq 0$. Suppose that the voltage across the thermistor is $\varphi_{0}$ in dimensionless terms, i.e., $V_{0} \varphi_{0}$ in dimensional terms. Then from (3.2) the dimensional current through 
the thermistor is

$$
I_{\mathrm{th}}=\pi \Gamma_{0}^{2} \sigma_{u} V_{0} \varphi_{0} /\left\{H \int_{0}^{1} \exp (f(u) / \varepsilon) d z\right\} .
$$

If the thermistor is "cold," i.e., $\gamma$ is not large enough for $u$ to reach the switching temperature $u=1, f(u)$ is small, and so

$$
I_{\mathrm{th}} \sim V_{0} \varphi_{0} \cdot \pi r_{0}^{2} \sigma_{u} / H
$$

i.e., there is a linear dependence of current on applied voltage. On the other hand, when the thermistor is "hot," and assuming that $\beta$ is small, the one-dimensional analysis predicts that

$$
I_{\mathrm{th}} \approx V_{0} \varphi_{0} \cdot \pi r_{0}^{2} \sigma_{u} / m \Delta,
$$

where $m \sim O(\varepsilon / \beta)$ is a constant depending on the precise details of the temperature profile. Since $\Delta=\gamma f^{\prime}\left(u^{*}\right) / \varepsilon, u^{*}=f^{-1}(\varepsilon \ln (\gamma / \varepsilon))$, and recalling that here we must use the value $\sigma_{u} \varphi_{0}^{2} V_{0}^{2} / 4 k \Delta T$ for $\gamma$, we have

$$
I_{0} \approx \frac{4 \pi k \varepsilon r_{0}^{2} \Delta T}{H m f^{\prime}\left(u^{*}\right)} \frac{1}{V_{0} \varphi_{0}} ;
$$

i.e., $I_{0}$ decreases with applied voltage. There is a transition region between these two regimes, but, in view of the exponential decay of $\sigma$ for $T>1$, it is rather small.

This current-voltage dependence is sketched in Fig. 7, which also shows the circuit law $I_{0}=\left(V_{0}-V_{0} \varphi_{0}\right) / R_{0}$, for various values of $V_{0}$. It is apparent that there may be one, two (exceptionally), or three operating points for a thermistor in a circuit, and that the current-voltage diagram for a thermistor in a circuit is as sketched in Fig. 8.

This nonuniqueness has been demonstrated theoretically in [4]. It is known to thermistor users [1] that when there are three operating points, the two extreme points ((a) and (c) in Fig. 7) are stable with (b) being unstable. It is an interesting open

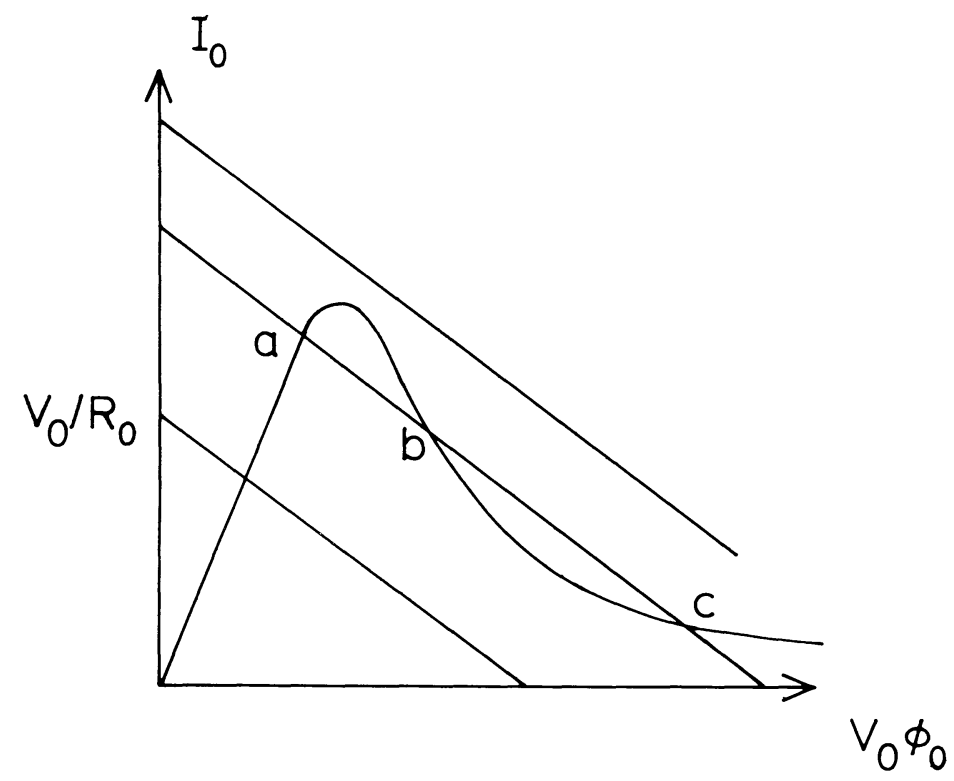

FIG. 7. Thermistor and circuit current characteristics versus the applied voltage. 


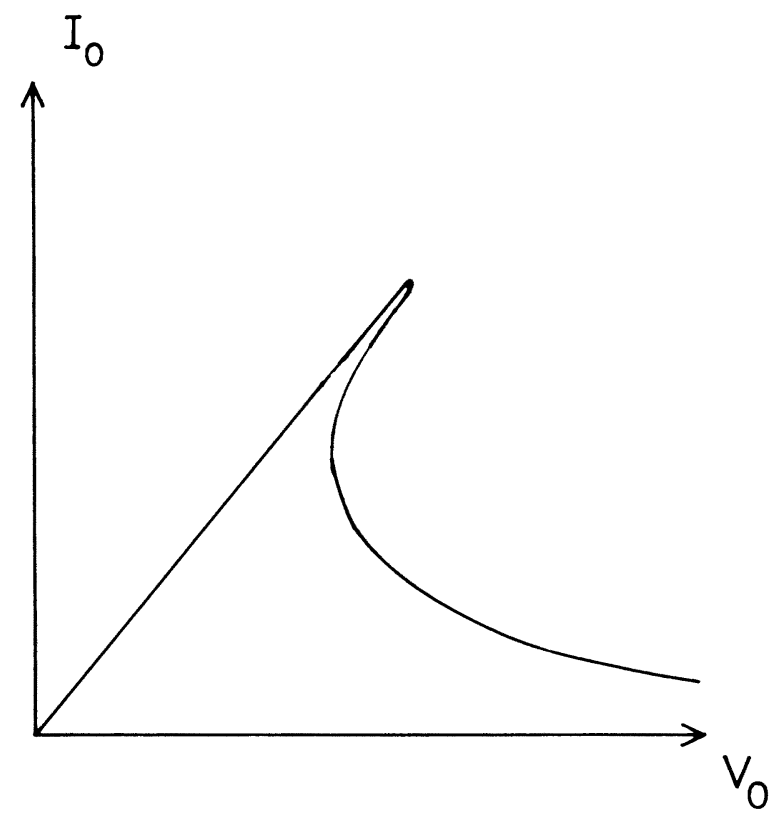

FIG. 8. Schematic diagram of current-voltage characteristic, showing a hysteretic switch between behaviour at low $V_{0}$ (cold) and high $V_{0}($ hot $)$.

problem to demonstrate which of these points is the large time limit of an initial value problem, although when used as a circuit protection device we would choose the parameters such that only point (c) remains.

4. We have predicted large temperature gradients during the temperature surges with spatial variation (see Fig. 4). These occur, in particular, if $\gamma / \mu^{2} \sim O(1)$. If the cracking mentioned in the Introduction is caused by thermal expansion, these large gradients are the most likely cause. They could be smoothed out by taking $\beta$ very small, but the operating temperature of the device might then become unacceptably large; alternatively, they may be suppressed at large values of $\gamma / \mu^{2}$, which, however, requires large voltages to be applied.

Acknowledgments. We thank all those who have participated in discussions on the thermistor, in particular, Dr. Miles Drake, then at STC, who brought this problem to Oxford, and also Colin Please, Rex Westbrook, John Ockendon, Andrew Lacey, Giovanni Cimatti, and Drs. R. Casselton and H. Macartney from STC. We also wish to acknowledge the contribution of John Hinch, who pointed out the possibility of obtaining an ordinary differential equation for the thermistor temperature (4.12) and who also showed numerically that the resulting prediction was quite accurate.

\section{REFERENCES}

[1] Anonymous, Philips Data Handbook on Components and Materials, II: Nonlinear Resistors, Philips Ltd., London, 1984.

[2] G. Cimatti, $A$ bound for the temperature in the thermistor problem, IMA J. Appl. Math., 40 (1988), pp. 15-22.

[3] - Remark on existence and uniqueness for the thermistor problem under mixed boundary conditions, Quart. Appl. Math., 47 (1989), pp. 117-121.

[4] - The stationary thermistor problem with a current limiting device, Proc. Roy. Soc. Edinburgh, 116A (1990), pp. 79-84. 
[5] G. Cimatti AND G. Prodi, Existence results for a nonlinear elliptic system modelling a temperature dependent electrical resistor, Ann. Mat. Pura Appl., 152 (1988), pp. 227-236.

[6] H. Diesselhorst, Uber das Probleme eines electrisch erwarmter Leiters, Ann. Phys., 1 (1900), pp. 312 325.

[7] S. D. Howison, Complex variables in industrial mathematics, in Proc. 2nd European Sympos. on Mathematics in Industry, Oberwolfach, Germany, H. Neunzert, ed., G. Teubner, Stuttgart, Germany, 1988, pp. 153-166.

[8] — A note on the thermistor problem in two space dimensions, Quart. Appl. Math., 47 (1989), pp. 509-512.

[9] S. D. Howison, J.-F. Rodrigues, And M. Shillor, Stationary solutions to the thermistor problem, J. Math. Anal. Appl., 1991, in press.

[10] A. A. LACEY, The spatial dependence of supercritical reacting systems, IMA J. Appl. Math., 27 (1981), pp. 71-84

[11] K. LuBITZ, Switching behaviour of power PTC resistors, Ber. Deutsche Keram. Ges., 55 (1978), pp. 322-324.

[12] G. MAdER, H. MEIXNER, AND P. KLEINSCHMIDT, Study of microscopic heat sources in semiconducting barium titanate ceramics, J. Appl. Phys., 56 (1984), pp. 2832-2836.

[13] H. OCKENDON AND J. R. OCKENDON, Variable-viscosity flows in heated and cooled channels, J. Fluid Mech., 83 (1977), pp. 177-190.

[14] J. R. A. PeArson, Variable-viscosity flows in channels with high heat generation, J. Fluid Mech., 83 (1977), pp. 191-206.

[15] R. WestbRoOK, The thermistor. A problem in heat and current flow, Numer. Methods Partial Differential Equations, 5 (1989), pp. 259-273. 